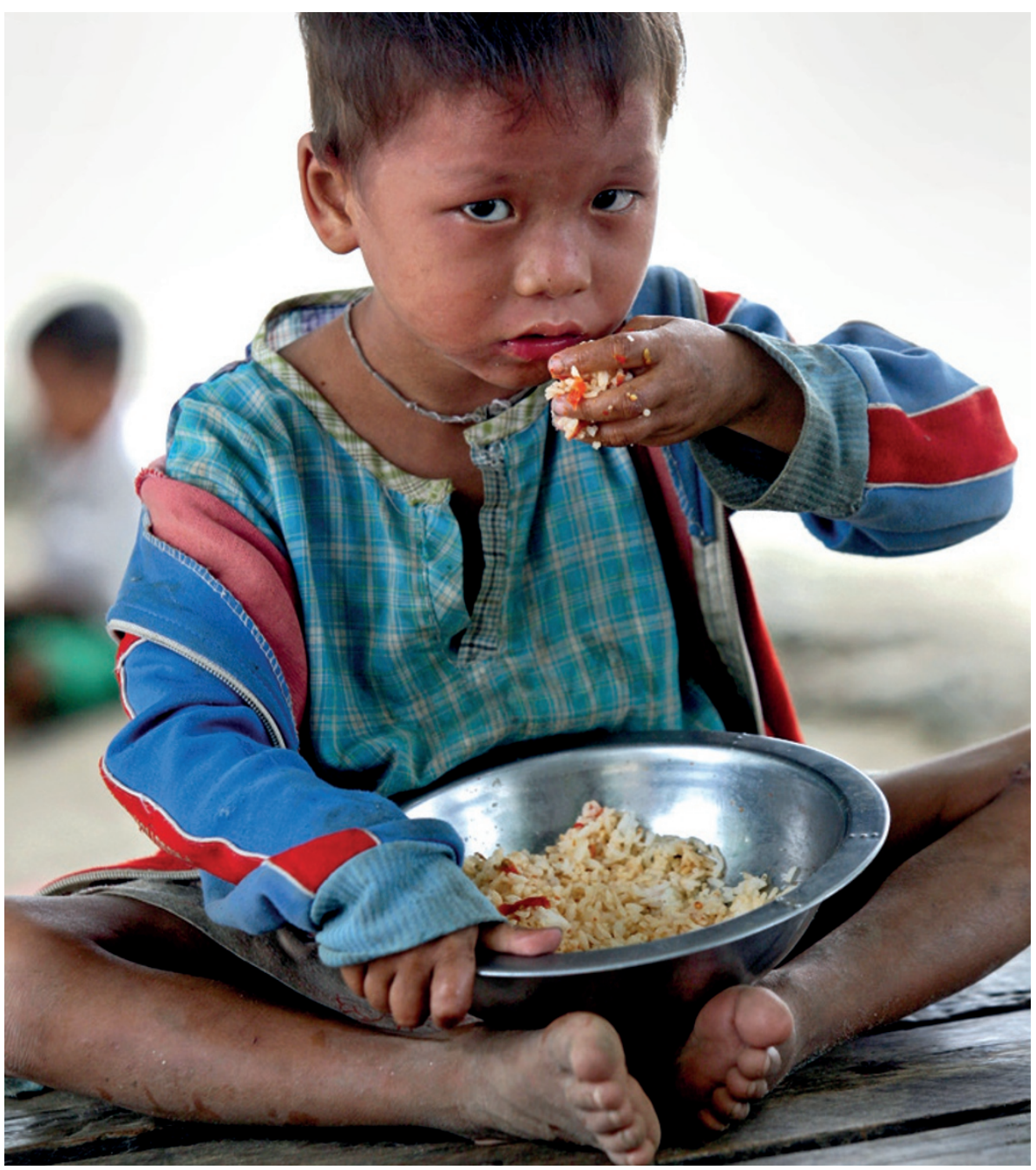

Rice is the main source of arsenic in food - it absorbs the metalloid more readily than other cereal grains.

CONTAMINATION

\title{
The toxic side of rice
}

\section{Around the world, researchers are looking for ways to rid rice of a troublesome companion.}

\section{BY EMILY SOHN}

$\mathrm{H}$ alf the world's population eats rice every day, making the grain a major source of nutrition for billions of people. But there is often something nasty in those grains alongside the vitamins, minerals and carbohydrates. Because of the way rice is grown, it can harbour arsenic, which is a threat to human health ${ }^{1}$.

"From all the work we've done over the years, it's quite plain that rice is the dominant source of inorganic arsenic in the human diet," says Andrew Meharg, a plant and soil scientist at Queen's University in Belfast, UK. "The cat's out of the bag. Now we have to do something about it."
The extent of the health risk is still unclear, but things are not looking good. Studies have linked chronic arsenic exposure with cancers of the bladder, lungs, skin and prostate, as well as heart disease. In the short term, it can cause gastrointestinal problems, muscle cramping and lesions on the hands and feet.

The risk of arsenic poisoning is greatest for people who eat rice several times a day, and for infants, whose first solid meals are often ricebased baby food.

In July 2014, the World Health Organization (WHO) set worldwide guidelines for what it considers to be safe levels of arsenic in rice, suggesting a maximum of 200 micrograms per kilogram for white rice and $400 \mu \mathrm{g} \mathrm{kg}^{-1}$ for brown rice.
The situation is especially dire in Bangladesh, where rice is the national staple and the water is naturally high in arsenic. Here as many as 100 million people suffer from acute arsenic poisoning from multiple sources.

The problem of contaminated rice is not limited to Asia. A 2012 study by the US-based advocacy group Consumers Union also found worrying levels of arsenic in rice sold in the United States. Some samples contained arsenic at more than twice the safe limit recommended by the WHO. The group suggested eating no more than two or three servings of rice each week. But eating less rice is not an option in many parts of the world where the food is an irreplaceable part of the culture, diet and lifestyle (see page $\mathrm{S} 50$ ).

At first glance, the problem may seem intractable. Farmers do not have access to arsenic-free water, and people need to eat rice, even if it is contaminated. But scientists hope that innovations in genetics, microbiology, agriculture and even cooking can break the cycle and keep arsenic out of one of the world's most important crops.

\section{MULTIPLE WHAMMY}

A variety of metals can accumulate in rice, including cadmium, lead and mercury. But arsenic (strictly a metalloid, not a metal) is the biggest problem, partly because it naturally occurs worldwide in soil and water ${ }^{2}$. It is especially common in the rocks of the Himalayas, from where the Ganges and other great rivers carry it to the heavily populated plains of south and southeast Asia. Most of the world's arsenic is locked up in mineral compounds underground, but mining and coal burning have released many tonnes into the environment (see 'Global arsenic cycle').

Rice is a particularly efficient scavenger of arsenic - it takes up ten times as much as other cereal grains - because it is the only grain traditionally grown in fields that are under water. Flooding makes soil conditions anaerobic, which causes arsenic to convert from bound and stable forms into more mobile ones.

In various states, arsenic has a similar chemical structure to phosphate and silicon, allowing it to sneak through the same pathways that plants use to absorb these important nutrients. Once inside, the arsenic becomes embedded in the roots, shoots, leaves and - particularly important for human health - the seeds. It accumulates most in the husk, the outer covering of the seed that is left intact in brown rice.

When rice is grown in an area with naturally high levels of arsenic, the problem becomes much worse. "It is a multiple whammy," says Shannon Pinson, a geneticist at the US Department of Agriculture's Dale Bumpers National Rice Research Center in Stuttgart, Arkansas. "It's in the soil and it's in the water that they're putting on the rice plants - and it's in the water they're cooking the rice in."

Some rice strains accumulate 20 -fold less arsenic than others. This suggests that certain 
$\sim$ varieties may have developed a way of blocking its uptake, offering hope that breeding could give the same powers to other strains.

Pinson and her colleagues studied more than 1,700 strains of rice from around the world ${ }^{3}$. They found that some varieties from the United States contained significantly less arsenic than other rice varieties grown using the same soil and water. Even more revealing, when they crossed some of these low-arsenic strains with high-arsenic varieties, exactly one fourth of the second-generation plants had low arsenic accumulation. Following the logic of simple Mendelian genetics, this finding suggests that a single gene is involved in the trait. However, the researchers have yet to figure out what gene it is, how it works, or even which of the plant's 12 chromosomes it can be found on. Other studies in Spain ${ }^{4}$ and the United States ${ }^{5}$ have flagged several genes and regions that may be involved in arsenic accumulation.

These and other findings raise the possibility of breeding arsenic resistance into strains from Bangladesh and other places where contamination is a major problem. Pinson says the idea of breeding low-arsenic rice is in its infancy, but "the best is yet to come".

\section{GENETIC INTERFERENCE}

Another way to prevent arsenic from accumulating in rice is to block its pathway from the roots to the grains. So far, researchers have discovered at least two types of transporter protein that carry the metalloid into the roots, says Barry Rosen, a molecular biologist at Florida International University's Herbert Wertheim College of Medicine in Miami. If scientists could identify the genes that govern the actions of these transporter proteins, they could potentially stop the flow of arsenic.

"We know some of the genetics, but I think there are many genes involved," says Yong-Guan Zhu, a biogeochemist at the Institute of Urban Environment at the Chinese Academy of Sciences in Beijing, who has collaborated with Rosen on several studies. "We are only exploring the tip of the iceberg."

Scientists hope that one day they can use genetic engineering to interfere with these arsenic-transport pathways, and there are many clever ideas for how that might work. Rosen and his group, for example, have created transgenic rice as well as transgenic soil microorganisms that both have the ability to turn arsenic into gas with the help of an extra enzyme originally discovered in algae that live in Yellowstone National Park ${ }^{6}$. Arsenic gas is not an ideal by-product, but Rosen says it would be quickly and safely diluted in the air. Some experimental rice strains have successfully released traces of arsenic in this way, but he says this is not yet efficient enough to be practical.

The prospect of genetic engineering opens the door for all sorts of big ideas. "If you could engineer rice to accumulate arsenic in the roots and not transfer it to the leaves and eventually the

\section{GLOBAL ARSENIC CYCLE}

Arsenic is a toxic element that is found in soil and water sources throughout the world. Arsenic can be found naturally in volcanic rock. Man-made sources include pesticides.

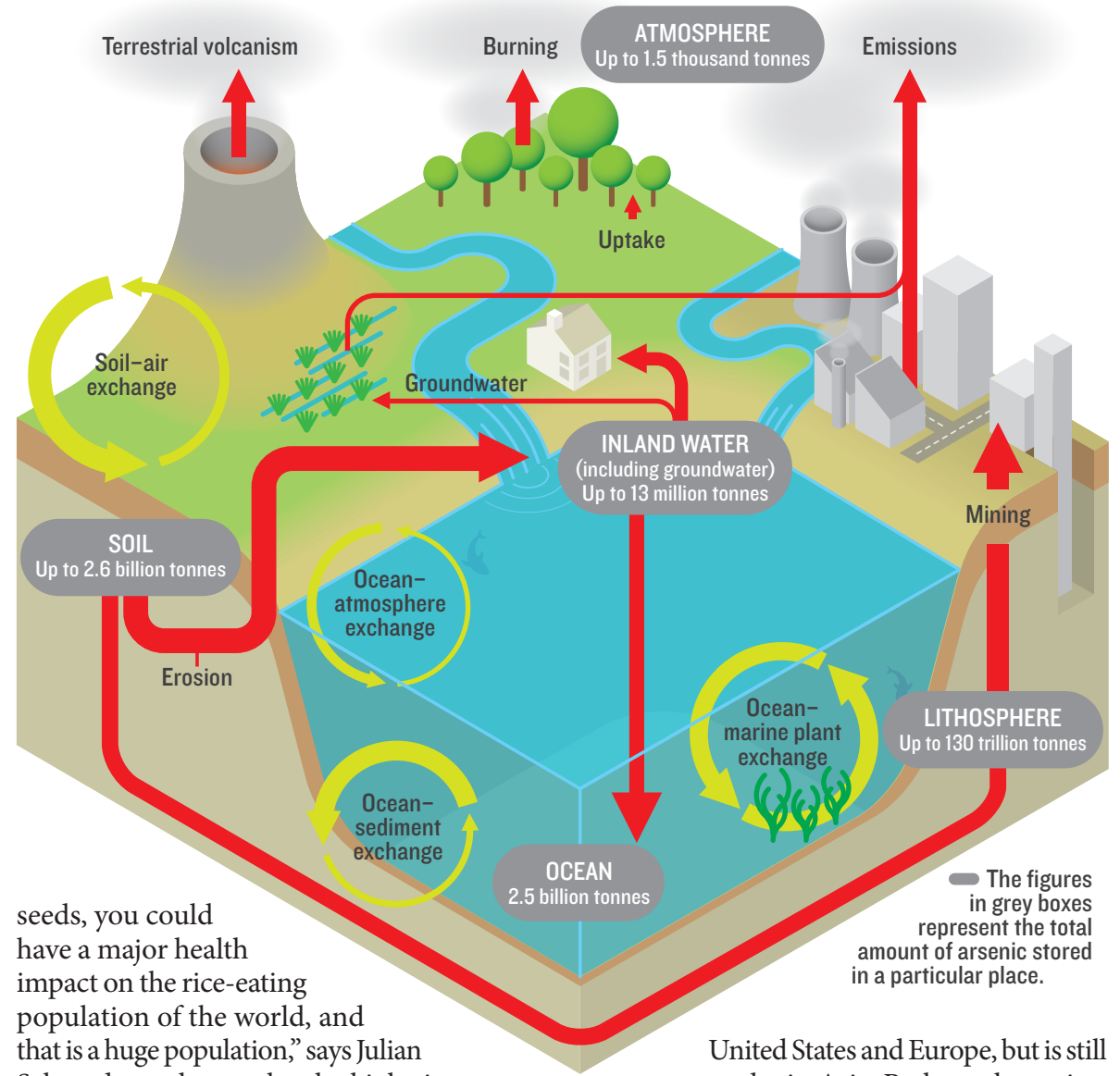
Schroeder, a plant molecular biologist at the University of California, San Diego. "I think it's very doable. It just hasn't been proven yet."

A different idea is to target the microorganisms that live in the soil and help plants access nutrients. One low-cost approach would be to inoculate soils with microbes that make arsenic less accessible to plants, says Janine Sherrier, a plant biochemist at the University of Delaware in Newark. Sherrier and her team have already identified one candidate bacterium, referred to as UD1023, that deposits a layer of iron around the roots, slowing the uptake of arsenic. "There's no one golden solution," she says. "You have to use all the tools at your disposal."

The way rice is grown and processed offers other opportunities for intervention. It is possible to cultivate the grain in dry soils that are moistened only by rain. This method, known as upland rice cultivation, reduces the arsenic load by a factor of 30 compared with traditional flooded rice paddies, Pinson says. But this approach is not possible in many waterlogged low-lying areas, including much of Bangladesh.

Milling - removing the husk and turning brown rice into white - also removes much of the arsenic, which accumulates in the outermost layers of the grain. As a result, brown rice contains 10- to 20-fold more arsenic than white, but it also contains many beneficial nutrients such as fibre and niacin. Brown rice is popular in the a novelty in Asia. Perhaps the easiest solutions of all lie in the kitchen. Instead of using equal parts water and rice when cooking, using three times more water than grain, and rinsing before and after cooking, can reduce the amount of arsenic by up to $30 \%$.

Meharg says that he has developed a way of contained in the rice grains. He cannot reveal details because the results have not yet been published, but he says the technique is simple and would require just a little low-cost engineering to create specialized cookers that would be affordable in even the poorest regions.

Anything that works is a welcome advance. "For the Chinese population, $60 \%$ of human arsenic exposure is from rice," Zhu says. "So if we can fix the rice problem, we can more or less fix arsenic exposure from food. It would make a huge difference."

Emily Sohn is a freelance science writer based in Minneapolis, Minnesota.

\footnotetext{
1. Meharg, A. et al. Env. Sci. Tech. 43, 1612-1617. (2009)

2. Zhu, Y. et al. Annu. Rev. Earth Planet.y Sci. 42, 443-467 (2014)

3. Pinson, S. R. M et al. Crop Sci. http://dx doi. org/10.2135/cropsci2013.10.0656 (2014).

4. Castrillo, G. et al. Plant Cell 25, 2944-2957 (2013).

5. Norton, G. et al. PLoS ONE 9, e89685 (2014).

6. Meng, X. et al. New Phyol. 191, 49-56 (2011)
} cooking rice that removes $80 \%$ of the arsenic 